\title{
ON THE MATTILA-SJÖLIN THEOREM FOR DISTANCE SETS
}

\author{
Alex Iosevich, Mihalis Mourgoglou and Krystal Taylor \\ University of Rochester, Department of Mathematics \\ Rochester, NY 14627, U.S.A.; iosevich@math.rochester.edu \\ UMR 8628 Université Paris-Sud 11-CNRS, Département de Mathématiques \\ Université Paris-Sud 11, F-91405 Orsay Cedex, France; mihalis.mourgoglou@math.u-psud.fr \\ University of Rochester, Department of Mathematics \\ Rochester, NY 14627, U.S.A.; taylor@math.rochester.edu
}

\begin{abstract}
We extend a result, due to Mattila and Sjölin, which says that if the Hausdorff dimension of a compact set $E \subset \mathbf{R}^{d}, d \geq 2$, is greater than $\frac{d+1}{2}$, then the distance set $\Delta(E)=\{\mid x-$ $y \mid: x, y \in E\}$ contains an interval. We prove this result for distance sets $\Delta_{B}(E)=\left\{\|x-y\|_{B}: x, y \in\right.$ $E$ \}, where $\|\cdot\|_{B}$ is the metric induced by the norm defined by a symmetric bounded convex body $B$ with a smooth boundary and everywhere non-vanishing Gaussian curvature. We also obtain some detailed estimates pertaining to the Radon-Nikodym derivative of the distance measure.
\end{abstract}

\section{Introduction}

The classical Falconer distance conjecture, originated in 1985, [2], says that if the Hausdorff dimension of a compact subset of $\mathbf{R}^{d}, d \geq 2$, is greater than $\frac{d}{2}$, then the Lebesgue measure of the set of distances, $\Delta(E)=\{|x-y|: x, y \in E\}$ is positive. In [2], Falconer proved the first result in this direction by showing that $\mathcal{L}^{1}(\Delta(E))>0$ if the Hausdorff dimension of $E$ is greater than $\frac{d+1}{2}$. See also [3] and [7] for a thorough description of the problem and related ideas. The best currently known results are due to Wolff in two dimensions, and to Erdogan [1] in dimensions three and greater. They prove that $\mathcal{L}^{1}(\Delta(E))>0$ if the Hausdorff dimension of $E$ is greater than $\frac{d}{2}+\frac{1}{3}$.

An important addition to this theory is due to Mattila and Sjölin [8] who proved that if the Hausdorff dimension of $E$ is greater than $\frac{d+1}{2}$, then $\Delta(E)$ not only has positive Lebesgue measure, but also contains an interval. This is accomplished by showing that the natural measure on the distance set has a continuous density. However, this set need not contain an interval with left end-point at the origin as illustrated by an example in ([7], p. 165). It was previously shown by Mattila [6] that if the ambient dimension is two or three, then the density of the distance measure is not in general bounded if the Hausdorff dimension of the underlying set $E$ is smaller than $\frac{d+1}{2}$. In higher dimensions, this question is still open for the Euclidean metric, but has been resolved if the Euclidean metric is replaced by a metric induced by a norm defined by a suitably chosen paraboloid. See [5].

doi:10.5186/aasfm.2012.3732

2010 Mathematics Subject Classification: Primary 28A75, 42B20, 52C10.

Key words: Falconer distance problem, Erdos problems, bilinear operators, distribution of angles, arithmetic of the lattice.

This work was partially supported by the NSF Grant DMS10-45404. 
In this paper we give an alternative proof of the Mattila-Sjölin result and extend it to more general distance sets $\Delta_{B}(E)=\left\{\|x-y\|_{B}: x, y \in E\right\}$, where $\|\cdot\|_{B}$ is the norm generated by a symmetric bounded convex body $B$ with a smooth boundary and everywhere non-vanishing Gaussian curvature.

Our main result is the following.

Theorem 1.1. Let $E$ be a compact subset of $\mathbf{R}^{d}, d \geq 2$, with Hausdorff dimension, denoted by $s$, greater than $\frac{d+1}{2}$. Let $\mu$ be a Frostman measure on E. Let $\sigma$ denote the Lebesgue measure on $\partial B$. Define the distance measure $\nu$ by the relation

$$
\int h(t) d \nu(t)=\iint h\left(\|x-y\|_{B}\right) d \mu(x) d \mu(y),
$$

where $\|\cdot\|_{B}$ is the norm generated by a symmetric bounded convex body $B$ with a smooth boundary and everywhere non-vanishing Gaussian curvature.

(i) Then the measure $\nu$ is absolutely continuous with respect to the Lebesgue measure.

(ii) We have

where

$$
\frac{\nu((t-\epsilon, t+\epsilon))}{2 \epsilon}=M(t)+R^{\epsilon}(t)
$$

$$
M(t)=\int|\widehat{\mu}(\xi)|^{2} \widehat{\sigma}(t \xi) t^{d-1} d \xi
$$

is the density of $\nu$ and

$$
\sup _{0<\epsilon<\epsilon_{0}}\left|R^{\epsilon}(t)\right| \lesssim \epsilon_{0}^{s-\frac{d+1}{2}}
$$

(iii) Moreover, $M \in C^{\left\lfloor s-\frac{d+1}{2}\right\rfloor}(I)$ for any interval I not containing the origin, where $\lfloor u\rfloor$ denotes the smallest integer greater than or equal to $u$. In particular, $M$ is continuous away from the origin if $s>\frac{d+1}{2}$ and therefore $\Delta_{B}(E)$ contains an interval in view of (i).

(iv) Suppose that $s>k+\alpha$, where $k$ is a non-negative integer and $0<\alpha<1$. Then the kth derivative of the density function of $\nu$ is Hölder continuous of order $\alpha$.

Remark 1.2. Metric properties of $\|\cdot\|_{B}$ are not used in the proof of Theorem 1.1. Let $\Gamma$ be a star shaped body in the sense that for every $\omega \in S^{d-1}$ there exists $1<r_{0}(\omega)<2$ such that $\left\{r \omega: 0 \leq r \leq r_{0}(\omega)\right\} \subset \Gamma$ and $\left\{r \omega: r>r_{0}(\omega)\right\} \cap \Gamma=\emptyset$. Define $\|x\|_{\Gamma}=\inf \{t>0: x \in t \Gamma\}$ and let $\Delta_{\Gamma}(E)=\left\{\|x-y\|_{\Gamma}: x, y \in E\right\}$. Let $\sigma_{\Gamma}$ denote the Lebesgue measure on the boundary of $\Gamma$. Then if $\left|\widehat{\sigma}_{\Gamma}(\xi)\right| \lesssim|\xi|^{-\frac{d-1}{2}}$, the conclusion of Theorem 1.1 holds with the same exponents.

1.1. Sharpness of results. As we note above, Mattila's construction [6] shows that if the Hausdorff dimension of $E$ is smaller than $\frac{d+1}{2}, d=2,3$, then the density of distance measure is not in general bounded in the case of the Euclidean metric. Moreover, Mattila construction can be easily extended to all metrics generated by a bounded convex body $B$ with a smooth boundary and non-vanishing Gaussian curvature.

In dimensions four and higher, all we know at the moment (see the main result in $[5])$ is that there exists a bounded convex body $B$ with a smooth boundary and nonvanishing curvature, such that the density of the distance measure is not in general 
bounded if the Hausdorff dimension of the underlying set $E$ is less than $\frac{d+1}{2}$. We do no know what happens when the Hausdorff dimension of $E$ equals $\frac{d+1}{2}$ in any dimension and for any smooth metric.

It would be very interesting if any of these results actually depended on the underlying convex body $B$ in a non-trivial way. This would mean that smoothness and non-vanishing Gaussian curvature of the level set do not tell the whole story. There is some evidence that this may be the case. See, for example, [4], where connections between problems of Falconer type and distribution of lattice points in thin annuli are explored.

If the Hausdorff dimension of $E$ is less than $\frac{d}{2}$, then the density of the distance measure, for any metric induced by a bounded convex body $B$ with a smooth boundary and non-vanishing curvature is not in general bounded by a construction due to Falconer [2].

Acknowledgements. The second named author holds a two-year Sophie Germain International post-doctoral scholarship in Fondation de Mathématiques Jacques Hadamard (FMJH) and would like to thank the faculty and staff of the Université Paris-Sud 11, Orsay for their hospitality.

\section{Proof of Theorem 1.1}

2.1. Proof of items (i) and (ii). The proof of item (i) of Theorem 1.1 is due to Falconer [2] and Mattila [6]. This brings us to item (ii). Recall that every compact set $E$ in $\mathbf{R}^{d}$, of Hausdorff dimension $s>0$ possesses a Frostman measure (see e.g. [7], p. 112), which is a probability measure $\mu$ with the property that given any $\delta>0$, for every ball of radius $r^{-1}$, denoted by $B_{r^{-1}}$, there exists $C_{\delta}>0$ such that

$$
\mu\left(B_{r^{-1}}\right) \leq C_{\delta} r^{-s+\delta} .
$$

Let

$$
\nu^{\epsilon}(t)=\frac{\nu((t-\epsilon, t+\epsilon))}{2 \epsilon}=\frac{1}{2 \epsilon} \mu \times \mu\left\{(x, y): t-\epsilon \leq\|x-y\|_{B} \leq t+\epsilon\right\} .
$$

We shall prove that $\lim _{\epsilon \rightarrow 0} \nu^{\epsilon}(t)$ exists and is a $C^{\left\lfloor s-\frac{d+1}{2}\right\rfloor}$ function.

Let $\rho$ be a smooth cut-off function, identically equal to 1 in the unit ball and vanishing outside the ball of radius 2 , with $\int \rho=1$. Let $\rho_{\epsilon}(x)=\epsilon^{-d} \rho(x / \epsilon)$. Since $\sigma_{t} * \rho_{\epsilon}$ is supported on the annulus of radius $t$ and width $\approx \epsilon$, and is $\approx \epsilon^{-1}$ on this annulus, there is no harm in working with the measure

$$
\iint \sigma_{t} * \rho_{\epsilon}(x-y) d \mu(x) d \mu(y)
$$

where $\sigma_{t}$ is the surface measure on the set $\left\{x:\|x\|_{B}=t\right\}$. By abusing notation slightly, we shall refer to this measure as $\nu^{\epsilon}(t)$.

By the Fourier inversion formula,

$$
\begin{aligned}
\nu^{\epsilon}(t) & =\int|\widehat{\mu}(\xi)|^{2} \widehat{\sigma}_{t}(\xi) \widehat{\rho}(\epsilon \xi) d \xi \\
& =\int|\widehat{\mu}(\xi)|^{2} \widehat{\sigma}_{t}(\xi) d \xi-\int|\widehat{\mu}(\xi)|^{2} \widehat{\sigma}_{t}(\xi)(1-\widehat{\rho}(\epsilon \xi)) d \xi=M(t)+R^{\epsilon}(t) .
\end{aligned}
$$


We shall prove that $M(t)$ is a $C^{\left\lfloor s-\frac{d+1}{2}\right\rfloor}$ function and that $\lim _{\epsilon \rightarrow 0} R^{\epsilon}(t)=0$. We start with the latter. We shall need the following stationary phase estimate. See, for example, [10], [9] or [11].

Lemma 2.1. Let $\sigma$ be the surface measure on a compact piece of a smooth convex surface in $\mathbf{R}^{d}, d \geq 2$, with everywhere non-vanishing Gaussian curvature. Then

$$
|\widehat{\sigma}(\xi)| \lesssim|\xi|^{-\frac{d-1}{2}}
$$

where here, and throughout, $X \lesssim Y$ means that there exists $C>0$ such that $X \leq C Y$. Moreover,

$$
\left|D^{\alpha} \widehat{\sigma}(\xi)\right| \leq C_{\alpha, d}|\xi|^{-\frac{d-1}{2}},
$$

where $D^{\alpha}$ is the differential operator with respect to the multi-index $\alpha=\left(\alpha_{1}, \ldots, \alpha_{d}\right)$.

We shall also need the following well-known estimate. See, for example, [3] and [7].

Lemma 2.2. Let $\mu$ be a Frostman measure on a compact set $E$ of Hausdorff dimension $s>0$. Then

$$
\int_{2^{j} \leq|\xi| \leq 2^{j+1}}|\widehat{\mu}(\xi)|^{2} d \xi \lesssim 2^{j(d-s)}
$$

and, consequently,

$$
\int|\widehat{\mu}(\xi)|^{2}|\xi|^{-\gamma} d \xi=c \iint|x-y|^{-d+\gamma} d \mu(x) d \mu(y) \lesssim 1
$$

if $\gamma>d-s$. Here, and throughout, $X \lesssim Y$, with the controlling parameter $r$ means that for every $\epsilon>0$ there exists $C_{\epsilon}>0$ such that $X \leq C_{\epsilon} r^{\epsilon} Y$.

To prove the lemma, observe that

$$
\int_{2^{j} \leq|\xi| \leq 2^{j+1}}|\widehat{\mu}(\xi)|^{2} d \xi \lesssim \int|\widehat{\mu}(\xi)|^{2} \psi\left(2^{-j} \xi\right) d \xi
$$

where $\psi$ is a suitable smooth function supported in $\left\{x \in \mathbf{R}^{d}: 1 / 2 \leq|x| \leq 4\right\}$ and identically equal to 1 in the unit annulus. By definition of the Fourier transform and the Fourier inversion theorem, this expression is equal to

$$
2^{d j} \iint \widehat{\psi}\left(2^{j}(x-y)\right) d \mu(x) d \mu(y) \lesssim 2^{j(d-s)}
$$

since $\widehat{\psi}$ decays rapidly at infinity.

By Lemma 2.1 and Lemma 2.2, we have

$$
\begin{aligned}
\left|R^{\epsilon}(t)\right| & \lesssim \int_{|\xi|>\frac{1}{\epsilon}}|\widehat{\mu}(\xi)|^{2}|\xi|^{-\frac{d-1}{2}} d \xi \\
& \leq \int_{|\xi|>\frac{1}{\epsilon}}|\widehat{\mu}(\xi)|^{2}|\xi|^{-\frac{d-1}{2}} d \xi=\sum_{j>\log _{2}(1 / \epsilon)} \int_{2^{j} \leq|\xi| \leq 2^{j+1}}|\widehat{\mu}(\xi)|^{2}|\xi|^{-\frac{d-1}{2}} d \xi \\
& \lesssim \sum_{j>\log _{2}(1 / \epsilon)} 2^{j(d-s)} 2^{-j \frac{d-1}{2}} \lesssim \epsilon^{s-\frac{d+1}{2}}
\end{aligned}
$$


and thus

$$
\sup _{0<\epsilon \leq \epsilon_{0}}\left|R_{\epsilon}(t)\right| \leq \epsilon_{0}^{s-\frac{d+1}{2}} .
$$

In order to handle $\left|R^{\epsilon}(t)\right|$ over the integral when $|\xi|<\frac{1}{\epsilon}$, we notice that $(1-\widehat{\rho}(\epsilon \xi))$ is 0 when $\xi=(0, \ldots, 0)$ and, by continuity, is small in a neighborhood of the origin. This calculation establishes all the claims in part ii) of Theorem 1.1.

We note that the weaker statement showing that $\lim _{\epsilon \rightarrow 0}\left|R_{\epsilon}(t)\right|=0$ follows in an easier way from the dominated convergence theorem.

2.2. Proof of item (iii). Once again, by Lemma 2.1, we have

$$
|M(t)| \lesssim \int|\widehat{\mu}(\xi)|^{2}|\xi|^{-\frac{d-1}{2}} d \xi
$$

and by the calculation identical to the one in the previous paragraph, we see that this quantity is $\lesssim 1$ if the Hausdorff dimension of $E$ is greater than $\frac{d+1}{2}$. Continuity follows by the Lebesgue dominated convergence theorem. The convergence of the integral allows us to differentiate inside the integral sign. We obtain

$$
M^{\prime}(t)=\int|\widehat{\mu}(\xi)|^{2} \frac{d}{d t}\left\{t^{d-1} \widehat{\sigma}(t \xi)\right\} d \xi .
$$

We have

$$
\frac{d}{d t}\left\{t^{d-1} \widehat{\sigma}(t \xi)\right\}=(d-1) t^{d-2} \widehat{\sigma}(t \xi)+t^{d-1} \nabla \widehat{\sigma}(t \xi) \cdot \xi .
$$

Applying (2.2) and (2.3) of Lemma 2.1 once more, it follows that

$$
\left|\frac{d}{d t}\left\{t^{d-1} \widehat{\sigma}(t \xi)\right\}\right| \lesssim|\xi|^{-\frac{d-1}{2}+1}
$$

Repeating the argument in 2.4, we see that $M^{\prime}(t)$ exists if the Hausdorff dimension of $E$ is greater than $\frac{d+1}{2}+1$. Proceeding in the same way one establishes that

$$
\frac{d^{m}}{d t^{m}}\left\{t^{d-1} \widehat{\sigma}(t \xi)\right\} \lesssim|\xi|^{-\frac{d-1}{2}+m}
$$

and the conclusion of Theorem 1.1 follows.

2.3. Proof of item (iv). We shall deal with the case $k=0$, as the other cases follow from a similar argument. Let

$$
\lambda(t)=t^{d-1} \widehat{\sigma}(t \xi)
$$

We must show that

$$
|M(u)-M(v)| \leq C|u-v|^{\alpha} .
$$

We have

$$
\begin{aligned}
M(u)-M(v) & =\int|\widehat{\mu}(\xi)|^{2}(\lambda(u)-\lambda(v)) d \xi \\
& =\int|\widehat{\mu}(\xi)|^{2}(\lambda(u)-\lambda(v))^{\alpha}(\lambda(u)-\lambda(v))^{1-\alpha} d \xi
\end{aligned}
$$

Now,

$$
\lambda(u)-\lambda(v)=(u-v) \lambda^{\prime}(c),
$$

where $c \in(u, v)$, by the mean-value theorem. It follows that

$$
|\lambda(u)-\lambda(v)|^{\alpha} \leq|u-v|^{\alpha}\left|\lambda^{\prime}(c)\right|^{\alpha} .
$$


On the other hand,

$$
|\lambda(u)-\lambda(v)|^{1-\alpha} \leq|\lambda(u)|^{1-\alpha}+|\lambda(v)|^{1-\alpha} .
$$

We have already shown above that

$$
|\lambda(u)| \lesssim|\xi|^{-\frac{d-1}{2}} \text { and }\left|\lambda^{\prime}(u)\right| \lesssim|\xi|^{-\frac{d-1}{2}+1} .
$$

It follows that

$$
|M(u)-M(v)| \lesssim|u-v|^{\alpha} \int|\widehat{\mu}(\xi)|^{2}|\xi|^{-\frac{d-1}{2}+\alpha} d \xi \lesssim|u-v|^{-\alpha}
$$

where the last step follows by Lemma 2.2, and so the item iv) follows.

\section{References}

[1] Erdo G̃an, B.: A bilinear Fourier extension theorem and applications to the distance set problem. - Int. Math. Res. Not. IMRN 2005:23, 2005.

[2] FALCONER, K. J.: On the Hausdorff dimensions of distance sets. - Mathematika 32, 1986, $206-212$.

[3] Falconer, K. J.: The geometry of fractal sets. - Cambridge Tracts in Math. 85, Cambridge Univ. Press, Cambridge, 1986.

[4] Iosevich, A., and M. Rudnev: Distance measures for well-distributed sets. - Discrete Comput. Geom. 38, 2007.

[5] Iosevich, A., and S. Senger: Sharpness of Falconer's estimate in continuous and arithmetic settings, geometric incidence theorems and distribution of lattice points in convex domains. arxiv.org/pdf/1006.1397 (submitted).

[6] Mattila, P.: On the Hausdorf dimension and capacities of intersections. - Mathematika 82, 1985, 213-217.

[7] Mattila, P.: Geometry of sets and measures in Euclidean spaces. - Cambridge Stud. Adv. Math. 44, Cambridge Univ. Press, Cambridge, 1995.

[8] Mattila, P., and Sjölin, P.: - Regularity of distance measures and sets. - Math. Nachr. 204, 1999, 157-162.

[9] Sogge, C.: Fourier integrals in classical analysis. - Cambridge Tracts in Math. 105, Cambridge Univ. Press, Cambridge, 1993.

[10] Stein, E. M.: Harmonic analysis. - Princeton Math. Ser. 43, Princeton Univ. Press, Princeton, NJ, 1993.

[11] Wolff, T.: Lectures on harmonic analysis. - Univ. Lecture Ser. 29, edited by I. Laba and C. Shubin, Amer. Math. Soc., Providence, RI, 2003.

Received 13 December 2011 